\title{
Pair-distribution functions of correlated composite fermions
}

\author{
Arkadiusz Wójs ${ }^{1,2}$, Daniel Wodziński ${ }^{1}$, and John J. Quinn ${ }^{2}$ \\ ${ }^{1}$ Wroclaw University of Technology, 50-370 Wroclaw, Poland \\ ${ }^{2}$ University of Tennessee, Knoxville, Tennessee 37996, USA
}

\begin{abstract}
Pair-distribution functions $g(r)$ of Laughlin quasielectrons (composite fermions in their second Landau level) are calculated in the fractional quantum Hall states at electron filling factors $\nu_{e}=4 / 11$ and 3/8. A shoulder in $g(r)$ is found, supporting the idea of cluster formation. The intra- and intercluster contributions to $g(r)$ are identified, largely independent of $\nu_{e}$. The average cluster sizes are estimated; pairs and triplets of quasielectrons are suggested at $\nu_{e}=4 / 11$ and $3 / 8$, respectively.
\end{abstract}

PACS numbers: 71.10.Pm, 73.43.-f

\section{INTRODUCTION}

Pan et al $1 \frac{1}{1}$ have recently observed the fractional quantum Hall (FQH) effect 2,3 in a spin-polarized twodimensional electron gas (2DEG) at the $\nu_{e}=\frac{4}{11}, \frac{3}{8}$, and $\frac{5}{13}$ fillings of the lowest Landau level (LL). In the composite fermion $(\mathrm{CF})$ model,,$\frac{4,5}{1}$ these values correspond to the fractional fillings $\nu=\frac{1}{3}, \frac{1}{2}$, and $\frac{2}{3}$ of the second CF LL, respectively. In Haldane's hierarchy picture ${ }^{6}$ of these states, Laughlin quasielectrons (QE's) fill (the same) fraction $\nu$ of their LL. The most striking conclusion from Pan's discovery is that the CF's (or QE's) can also form incompressible states when partially filling a LL. This could not be predicted by a simple analogy with known fractional electron liquids (Laughlin,,$\frac{3}{\underline{-}}$ Jain, 4 or Moore- $\operatorname{Read}^{7}$ states), because of a different form of $\mathrm{QE}-\mathrm{QE}$ interaction, $\stackrel{8.9 .10}{ }$ therefore yielding qualitatively different QE-QE correlations.

Although several numerical studies of interacting QE's have been reported $10,11,12,13$ and ideas such as CF flavormixing, ${ }^{14} \mathrm{QE}$ pairing, $\stackrel{15,16}{ }$ or stripes ${ }^{17}$ were invoked, the correlations responsible for the FQHE at $\nu_{e}=\frac{4}{11}$ and $\frac{3}{8}$ are not yet understood. It has not even been settled if these FQH states are isotropic, and the energies of liquid and solid phases were compared recently ${ }^{20}$ (although the Laughlin form was arbitrarily assumed for the liquid).

Sometimes overlooked is a general connection ${ }^{18,19}$ between the form of Haldane pseudopotential, ${ }^{21}$ occurrence of Laughlin correlations, and the validity of CF transformation. Actually, the form of QE-QE interaction is known from independent calculations, 8,9,10 and Laughlin correlations among the QE's have been ruled out using both a general pseudopotential argument ${ }^{9}$ and a direct analysis of many-QE wavefunctions ${ }^{12}$ In this paper we refer to the following well-established facts:

(i) The QE-QE Haldane pseudopotential21 is known from exact diagonalization of the Coulomb interaction among electrons in the lowest LL 8.9 .10 Since there are no unchecked assumptions in such a calculation, it must be regarded a "numerical experiment." Neither finitesize errors, lowest-LL restriction, finite 2DEG width, nor other details of realistic experimental systems affect the dominant feature of the pseudopotential which is the lack of strong $Q E-Q E$ repulsion at short range. (ii) The QE's do not ${ }^{9.12}$ have Laughlin correlations at $\nu=\frac{1}{3}$ corresponding to $\nu_{e}=\frac{4}{11}$. The Moore-Read halffilled state is not ${ }^{12.22}$ an adequate description of QE-QE correlations at $\nu=\frac{1}{2}$ corresponding to $\nu_{e}=\frac{3}{8}$.

(iii) A sequence of nondegenerate finite-size QE ground states with a gap, extrapolating to $\nu=\frac{1}{3}$ has been found $^{12}$ on a sphere. Although spherical geometry is not adequate for studying crystal or other broken-symmetry phases, the identified states appear incompressible and have the lowest energy of all QE liquids (considerably below the Laughlin state).

To address the problem of correlations at $\nu_{e}=\frac{4}{11}, \frac{3}{8}$, and $\frac{5}{13}$ we calculate pair-distribution functions $g(r)$ in the incompressible liquid ground states of up to $N=14$ QE's. Their comparison with the (known) curves of the Laughlin and Moore-Read states implies a different nature of the QE correlations in these novel FQH states. It shows that their incompressibility cannot be explained by a simple analogy between the QE and electron liquids, and suggests that different wavefunctions need be proposed for the correlated CF's. Unfortunately, the calculated $g(r)$ are of little help in a precise definition of these wavefunctions, even though some qualitative statements can be made about the QE correlations.

From our finite-size results we identify and analyze the size-independent features in $g(r)$ : the $\sim r^{2}$ behavior at short range and a shoulder at a medium range, and argue that they are consistent with the idea 12 of QE cluster formation. Short- and long-range contributions to $g(r)$ are found, describing correlations between the QE's from the same or different clusters. Both intra- and intercluster QE-QE correlations depend rather weakly on $\nu$. The average size of the clusters is estimated; it seems that the QE's form pairs at $\nu=\frac{1}{3}$ and triplets at $\nu=\frac{1}{2}$. A similar analysis of $g(r)$ carried out for the Moore-Read state reveals a qualitatively different behavior.

\section{MODEL}

\section{A. Haldane sphere}

The numerical calculations have been carried out in Haldane's spherical geometry, ${ }^{6}$ convenient for the exact 
study of short-range correlations. In this model, the lowest LL for particles of charge $q$ is a degenerate shell of angular momentum $l=Q$. Here $2 Q$ is the strength of Dirac monopole in the center of the sphere defined in the units of elementary flux $\phi_{0}=h c / q$ as $2 Q \phi_{0}=4 \pi R^{2} B$, the total flux of the magnetic field $B$ through the surface of radius $R$. Using the usual definition of the magnetic length, $\lambda=\sqrt{\hbar c / q B}$, this can be written as $l \lambda^{2}=R^{2}$. In the following, $\lambda$ denotes the $\mathrm{QE}$ magnetic length corresponding to the fractional charge $q=-e / 3$.

The relative $(\mathcal{R})$ and total $(L)$ pair angular momenta are related via $L=2 l-\mathcal{R}$. For fermions, $\mathcal{R}$ is an odd integer, and it increases with increasing average pair separation $\sqrt{\left\langle r^{2}\right\rangle}$. The interaction (within the lowest LL) is entirely determined by Haldane pseudopotential defined as the pair interaction energy $V$ as a function of $\mathcal{R}$.

\section{B. Exact diagonalization}

Recently, we have identified ${ }^{12}$ the series of finite-size spin-polarized states that in the thermodynamic limit describe the FQHE at $\nu_{e}=\frac{4}{11}$ and $\frac{3}{8}$. To do so, we have carried out extensive exact-diagonalization calculations for interacting QE's (particles in the second CF LL). On Haldane sphere, $N$ fermionic QE's were confined in a standard way to an angular momentum shell of degeneracy $\Gamma=2 l+1$, corresponding to the QE filling factor $\nu \sim N / \Gamma$, and the Haldane QE-QE pseudopotential $V(\mathcal{R})$ was taken from earlier calculations $\underline{8.9 .10}$

Regardless of the electron layer width $w$, magnetic field $B$, or other experimental parameters, the dominant feature of $V(\mathcal{R})$ is strong repulsion at $\mathcal{R}=3$. This feature alone determines the wavefunctions at $\frac{1}{3} \leq \nu \leq \frac{1}{2}$ (with the $\mathrm{QE}-\mathrm{QE}$ correlations consisting of maximum possible avoidance of Haldane pair amplitude $\mathcal{G}$ at $\mathcal{R}=3$ ), which are hence virtually insensitive to the (sample-dependent) details of $V(\mathcal{R})$. This justifies model calculations using $V(\mathcal{R})$ of Refs. 8 9 . Actually, a model pseudopotential as simple as $V=\delta_{\mathcal{R}, 3}$ is sufficient to reproduce correct correlations and incompressibility at $\nu_{e}=\frac{4}{11}$ or $\frac{3}{8}$.

\section{NUMERICAL RESULTS}

\section{A. Energy spectra}

The numerical results carried out for $N \leq 14$ (two sample spectra are displayed in Fig. 1) showed ${ }^{12}$ a sequence of nondegenerate (i.e., at the total angular momentum $L=0)$ ground states at $2 l=N / \nu-\gamma$ with $\nu=\frac{1}{3}$ and $\gamma=7$. The significant and well-behaved (as a function of $N$ ) excitation gap along this sequence strongly suggests that it represents the infinite $\nu_{e}=\frac{4}{11} \mathrm{FQH}$ state observed in experiment $\frac{\underline{\underline{1}}}{\mathrm{~T}}$ The value $\gamma \neq 3$ precludes Laughlin correlations among QE's in this state (earlier ruled out indirectly, based on the form of $\mathrm{QE}-\mathrm{QE}$ pseudopotentia $\left.{ }^{9}\right)$,

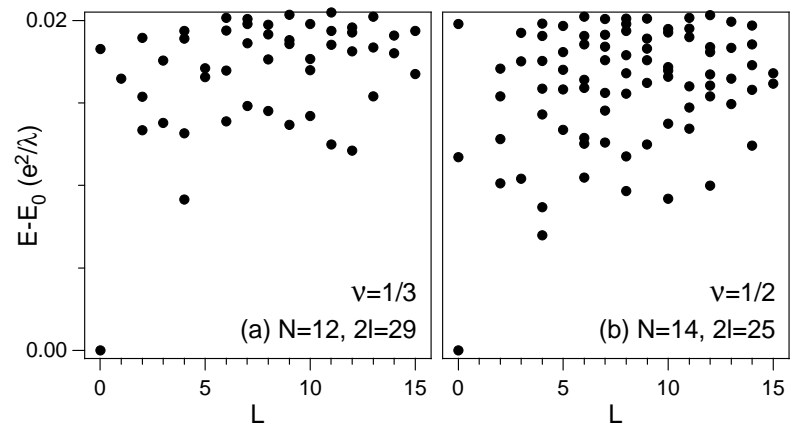

FIG. 1: Excitation energy spectra (energy $E$ as a function of total angular momentum $L ; E_{0}$ is the ground state energy) of $N$ interacting QE's on a sphere, at the values of CF LL degeneracy $\Gamma=2 l+1$ corresponding to the incompressible ground states at the QE filling factors $\nu=\frac{1}{3}$ (a) and $\frac{1}{2}$ (b).

i.e., an idea that the $\nu_{e}=\frac{4}{11}$ state is simply a Haldane hierarchy state of Laughlin-correlated CF's. While the exact correlations in this (known only numerically for a few consecutive $N$ ) ground state have not yet been defined, their vanishing degeneracy $(L=0)$ implies that they describe a QE liquid, rather than a broken-symmetry state (such as liquid-crystal nematic states proposed ${ }^{23}$ in the context of FQHE at different values of $\nu$ ).

Another sequence was anticipated at $2 l=2 N-\gamma$ to represent the infinite $\nu_{e}=\frac{3}{8} \mathrm{FQH}$ state. However, the only ground state with a significant gap and remaining outside of the $\nu=\frac{1}{3}$ sequence (or its particle-hole symmetric $\nu=\frac{2}{3}$ sequence at $\left.2 l=\frac{3}{2} N+2\right)$ occurs 12 for $N=14$ and $2 l=25$ (and it also has $L=0$ ). These values of $(N, 2 l)$ happen to belong to a $2 l=2 N-3$ series representing the Moore-Read (pfaffian) paired state, but the overlap between the two turns out nearly zero. 12.22 Moreover, the ground states for the two neighboring even (as appropriate for a hypothetically paired state) values of $N=12$ and 16 (and $2 l=21$ and 29) have $L>0$ and no gap, the value of $2 l=17$ for $N=10$ coincides with the $\nu=\frac{2}{3}$ sequence (so that only for $N>8$ can the filling factor $\nu$ be meaningfully assigned), and we are unable to compute the spectra for $N \geq 18$. Nevertheless, despite little evidence available from numerical diagonalization, the ground state for $N=14$ and $2 l=25$ (and its particle-hole counterpart at $N=12$ and the same $2 l=25)$ may possibly represent the $\nu_{e}=\frac{3}{8} \mathrm{FQH}$ state (i.e., have similar correlations causing incompressibility).

\section{B. Pair-distribution functions}

The QE-QE pair-distribution functions $g(r)$ have been calculated for the incompressible many-QE ground states as expectation values of the appropriate pair interaction,

$$
g(r)=(2 / N)^{2}\langle\delta(R \theta-r)\rangle .
$$

Here, $\theta$ is the relative angle on a sphere, so that $r$ measures interparticle distance along the surface (rather than 


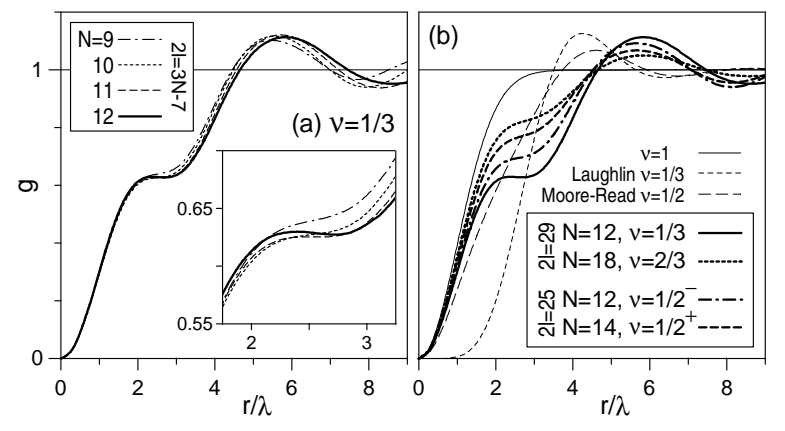

FIG. 2: QE-QE pair-distribution functions $g(r)$ of the incompressible ground states at different QE filling factors $\nu$. (a) curves for $\nu=\frac{1}{3}$ and different QE numbers $N$; (b) curves for QE's at different $\nu$ (thick lines) compared to some known incompressible states of electrons.

chord distance). More accurately, $r$ is the distance between the centers of extended QE's (note that in the calculation of many-QE wavefunctions, the system of QE's is mapped onto the lowest LL of point charges interacting through an effective pseudopotential). The prefactor in Eq. (11) ensures proper normalization, $g(\infty) \rightarrow 1$. Denoting infinitesimal area by $d S=2 \pi R^{2} d(\cos \theta)$ or (in magnetic units) by $d s=d S / 2 \pi \lambda^{2}$, we get an equivalent normalization condition,

$$
\int[1-g(r)] d s=\frac{2 l}{N} \rightarrow \nu^{-1}
$$

in large systems. Since $d s=l d(\cos \theta)$, a "local filling factor" can also be defined as $\nu(r)=d N / d s=(N / 2 l) g(r)$, and it satisfies $\nu(\infty)=\nu$ and $\int \nu(r) d s=N-1$.

The results for the $\nu=\frac{1}{3}$ sequence at $2 l=3 N-7$ are shown in Fig. 2(a). Similarity of all four curves is evident, indicating size-independent form of correlations (hence, describing an infinite system), with a welldeveloped shoulder around $r \approx 2.5 \lambda$. Similar shoulders occur in $g(r)$ of all incompressible ground states at $\nu=\frac{2}{3}$ or $\frac{1}{2}$ (the $\nu=\frac{2}{3}$ sequence at $2 l=\frac{3}{2} N+2$ is obtained from $2 l=3 N-7$ by replacing $N$ with $\Gamma-N$, while at $\nu=\frac{1}{2}$ there are two particle-hole conjugate sequences at $2 l=2 N-3$ and $2 N+1$, denoted by $\nu=\frac{1}{2}^{ \pm}$). The four curves representative of $\nu=\frac{1}{3}, \frac{2}{3}$, and $\frac{1}{2}^{ \pm}$are shown in Fig. 2(b). They are all clearly different from those marked with thin lines and describing correlations known for other incompressible FQH states (full LL, Laughlin $\nu=\frac{1}{3}$ state, or Moore-Read half-filled state). This is a direct indication of a different nature of $\mathrm{QE}-\mathrm{QE}$ correlations responsible for the FQHE at $\nu_{e}=\frac{4}{11}$ and $\frac{3}{8}$.

Let us stress that although the $\mathrm{QE}-\mathrm{QE}$ interactions are not known with great accuracy, the correlation functions in Fig. 2] are rather insensitive to the details of $V(\mathcal{R})$, as long as the dominant repulsion occurs at $\mathcal{R}=3$ (which seems to be universally true in the systems studied experimentally). This insensitivity is reminiscent of the Laughlin wavefunction, which also very accurately de-

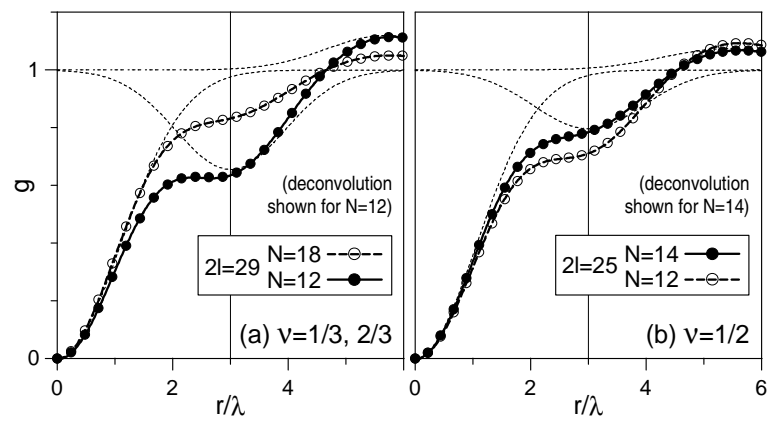

FIG. 3: Gaussian deconvolution of the QE-QE pair distribution functions $g(r)$ : dots - data of Fig. 2(b); lines - fits.

TABLE I: Gaussian deconvolution parameters for QE-QE pair-distribution functions shown in Figs. 2(b) and 3]

\begin{tabular}{c|ccc|ccc|ccc}
\hline \hline$\nu$ & $A_{0}$ & $\delta_{0}$ & $\sigma_{0}$ & $A_{1}$ & $\delta_{1}$ & $\sigma_{1}$ & $A_{2}$ & $\delta_{2}$ & $\sigma_{2}$ \\
\hline $1 / 3$ & 1 & 0 & 1.0989 & 0.3450 & 3 & 0.9412 & -0.1199 & 5.6905 & 1.0298 \\
$2 / 3$ & 1 & 0 & 1.0419 & 0.1535 & 3 & 0.9361 & -0.0530 & 5.6655 & 0.9987 \\
$1 / 2^{+}$ & 1 & 0 & 1.0626 & 0.2034 & 3 & 0.9475 & -0.0741 & 5.4041 & 1.1011 \\
$1 / 2^{-}$ & 1 & 0 & 1.0896 & 0.2755 & 3 & 0.9431 & -0.1005 & 5.4156 & 1.0903 \\
\hline \hline
\end{tabular}

scribes the actual $\nu=\frac{1}{3}$ ground state for a wide class of electron-electron pseudopotentials. However, while the avoidance of $\mathcal{R}=1$ by the electrons in the lowest LL can be elegantly described by flux attachment in the CF picture, no similar model has been proposed yet for the avoidance of $\mathcal{R}=3$ by the QE's. Therefore, knowing the $g(r)$ curves of QE's and understanding their correlations, we still cannot write their wavefunctions.

\section{Gaussian deconvolution}

The curves of Fig. 2(b) can be accurately deconvoluted using gaussians, $G(r / \lambda)=A \exp \left[-(r / \lambda-\delta)^{2} / 2 \sigma^{2}\right]$. This is shown in Fig. 3 where the symbols mark the exact data of Fig. 2(b) and the lines give the (nearly perfect) fits using three gaussians, $g=1-G_{0}-G_{1}-G_{2}$ (sufficient for $r \leq 6 \lambda)$. The fitted values of $\left[A_{i}, \delta_{i}, \sigma_{i}\right]$ for all four curves are listed in Tab. I Note that $A_{0}=1, \delta_{0}=0$, and $\delta_{1}=3$ for all curves (the latter value being least obvious, but probably resulting from the avoidance of the same $\mathcal{R}_{3}=3$ by the QE's at all values of $\nu$ ). The values of the $G_{2}$ parameters are not very meaningful when the next term in the approximation $\left(G_{3}\right)$ is neglected. The clearest difference between the four curves is in $A_{1}$.

\section{Short/long-range deconvolution}

It appears more physically meaningful to decompose $g(r)$ into $g_{0}=1-\exp \left(-r^{2} / 2 \lambda^{2}\right)$ describing a full lowest 


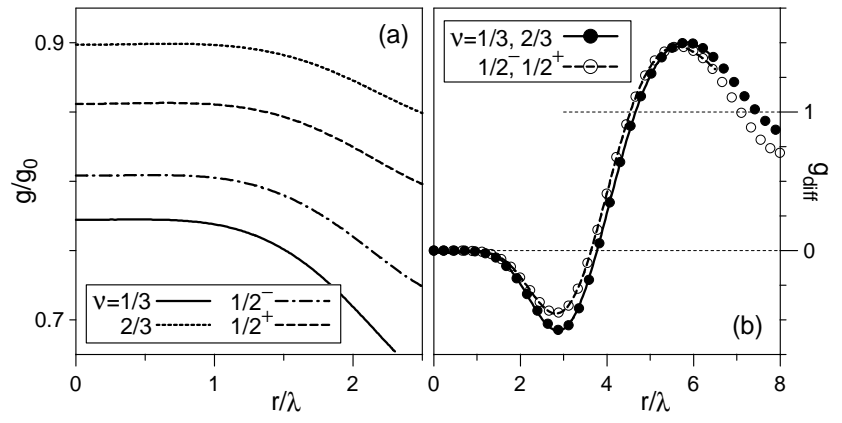

FIG. 4: (a) Ratio of QE-QE pair-distribution functions $g(r)$ to $g_{0}(r)$ of a full lowest LL for different incompressible QE ground states; (b) the "remainder" $g_{\text {diff }}(r)$ defined by Eq. (3).

$\mathrm{LL}^{24}$ and a (properly normalized) "remainder" $g_{\text {diff }}$,

$$
g(r)=\alpha g_{0}(r)+(1-\alpha) g_{\mathrm{diff}}(r) .
$$

For each $g(r)$, parameter $\alpha$ is calculated as the limit of $g / g_{0}$ at $r \rightarrow 0$. It is clear from Fig. 世 a) that $g(r)$ is accurately approximated by $\alpha g_{0}(r)$ within a finite area or a radius $\sim \lambda$ for all four ground states of Fig. 2(b). The numerical values of $\alpha$ are $0.772,0.804,0.856$, and 0.899 for $\nu=\frac{1}{3}_{3} \frac{1}{2}^{-}, \frac{1}{2}^{+}$, and $\frac{2}{3}$, respectively. Evidently, $\alpha$ is size-dependent (e.g., the pair of values for $\nu=\frac{1}{2}^{ \pm}$ must converge to the same thermodynamic limit).

The four curves $g_{\text {diff }}(r)$ calculated from Eq. (33) are plotted in Fig. 目(b). Symbols and lines mark the exact data and the three-gaussian fits of Tab. I respectively. We note that: (i) For the pairs of particle-hole conjugate states $(N=12,18$ at $2 l=29$ and $N=12,14$ at $2 l=25)$, the $g_{\text {diff }}(r)$ curves are identical. (ii) The curves obtained for $\nu=\frac{1}{3}$ and $\frac{1}{2}$ are very similar (and possibly identical in large systems); they all vanish at short range and have a minimum at $r \approx 3 \lambda$ and a maximum at $r \approx 5.5 \lambda$.

\section{DISCUSSION}

\section{A. QE clustering}

Some information about the form of QE-QE correlations can be easily deduced from the form of interaction pseudopotential $V(\mathcal{R})$, which is simply the interaction hamiltonian defined only for those pair states allowed in the lowest LL. In low-energy many-body states the particles generally tend to avoid pair eigenstates with high interaction energy, which means minimization of the corresponding Haldane pair amplitude $\mathcal{G}$. If repulsion $V$ decreases sufficiently quickly $\frac{18}{}$ as a function of $\mathcal{R}$ (the exact criterion being ${ }^{19}$ that $V$ decreases sublinearly as a function of $\sqrt{\left\langle r^{2}\right\rangle}$, the smallest value of $\mathcal{R}=1$ is avoided. This Laughlin type of correlation is elegantly described by attachment of $2 p=2$ fluxes to each particle in the $\mathrm{CF}$ transformation. In a Laughlin-correlated state, each particle avoids being close to any other particle (as much as possible at a given finite $\nu$ ).

When short-range repulsion weakens ( $V$ at $\mathcal{R}=1$ decreases compared to $V$ at $\mathcal{R} \geq 3$ ), Laughlin correlations disappear and can be replaced by pairing or formation larger clusters. Pairs 15.16 or clustering ${ }^{12}$ were suggested by several authors for the QE's. This idea was justified by an observation that QE-QE pseudopotential nearly vanishes at $\mathcal{R}=1$ and is strongly repulsive at $\mathcal{R}=3$, causing an increase of $\mathcal{G}(1)$ and a simultaneous is decrease of $\mathcal{G}(3)$ compared to the Laughlin-correlated state ${ }^{12}$

The assumption that QE's form clusters naturally explains a shoulder in $g(r)$, and allows one to interpret $g_{0}$ and $g_{\text {diff }}$ as the intra- and inter-cluster QE-QE correlations, i.e. the short- and long-range contributions to $g$, corresponding to the $\mathrm{QE}$ pairs belonging to the same or different clusters, respectively. The vanishing of $g_{\text {diff }}(r)$ at short-range reflects isolation of QE's belonging to different clusters. The reason why $g_{\text {diff is not positive }}$ definite is that intra-cluster correlations are accurately described by $g_{0}$ only within a certain radius. In other words, the actual inter-cluster contribution to $g$ is not $e x$ -

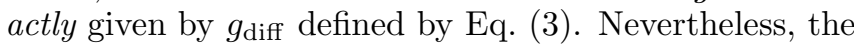
following two conclusions remain valid: (i) the intra- and inter-cluster QE-QE correlations are similar at $\nu=\frac{1}{3}, \frac{1}{2}$, and $\frac{2}{3}$, with the respective correlation-hole radii $\varrho_{0} \sim \lambda$ and $\varrho_{1} \sim 4 \lambda$; and (ii) the cluster size $K$ depends on $\nu$.

Similar form of $g(r)$ was found ${ }^{23}$ for broken-symmetry Laughlin states, in which the shoulder results from angular averaging of an anisotropic function $g(r, \phi) \sim r^{2}$ or $r^{6}$, depending on $\phi$. However, the present case of QE's is different, because $g(r)$ is isotropic (wavefunctions have $L=0$ ) and the shoulders result from radial averaging of inter- and intra-cluster correlations, (beginning as $\sim r^{2}$ and a higher power of $r$ at short range, respectively).

\section{B. Average cluster size}

In a clustered state, the (average) cluster size $K$ is connected to $\alpha$, and the form of $g_{\text {diff }}$ depends on correlations between the clusters. The values of $K$ at $\nu=\frac{1}{3}$ or $\frac{1}{2}$ can be estimated by comparison of the actual parameters $\alpha$ with those predicted for the hypothetical states of $N$ particles arranged into $N / K$ independent $K$-clusters. By independence of the clusters we mean that inter-cluster correlations do not affect the local filling factor $\nu(r)$ at short range. For a single cluster, which on a sphere is the $K$-particle state with the maximum total angular momentum $L=K l-\frac{1}{2} K(K-1)$, the $\nu_{K}(r)$ depends on the surface curvature and thus (through $R / \lambda=\sqrt{l}$ ) on $2 l$.

We have calculated the prefactors $\beta_{K}$ of the shortrange approximation $\nu_{K}(r) \approx \beta_{K} g_{0}(r)$ for different values of $K$ and $2 l$ and listed some in Tab. III (note that $\nu_{2}(r)$ is known exactly). These coefficients are to be compared with $\beta=(N / 2 l) \alpha$ of the incompressible $N$ QE states obtained from diagonalization. Of course, this approach is somewhat questionable as one generally can- 
TABLE II: Parameters $\beta_{K}$ of the short-range approximation $\nu(r) \sim \beta g_{0}(r)$ obtained for independent clusters of size $K$.

\begin{tabular}{c|ccccc}
\hline \hline $2 l$ & $\beta_{2}$ & $\beta_{3}$ & $\beta_{4}$ & $\beta_{5}$ & $\beta_{6}$ \\
\hline 25 & 0.2768 & 0.4196 & 0.5110 & 0.5765 & 0.6269 \\
29 & 0.2730 & 0.4134 & 0.5029 & 0.5669 & 0.6159 \\
60 & 0.2609 & 0.3938 & 0.4778 & 0.5372 & 0.5821 \\
$\infty$ & 0.2500 & 0.3763 & 0.4555 & 0.5110 & 0.5527 \\
\hline \hline
\end{tabular}

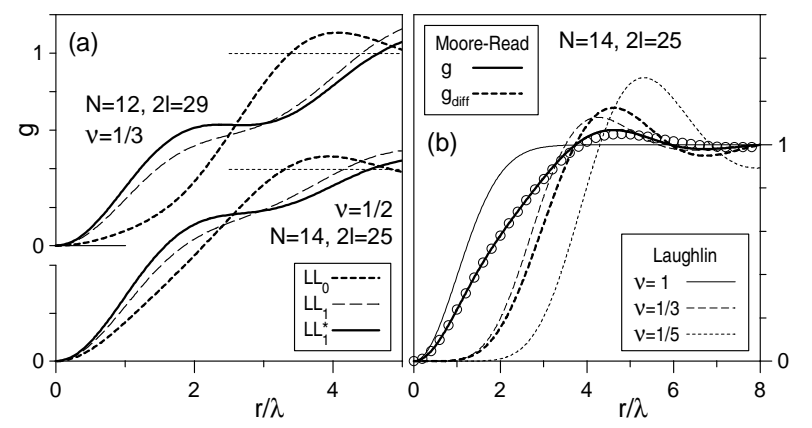

FIG. 5: (a) Pair-distribution functions $g(r)$ of lowest $L=0$ states of finite systems corresponding to $\nu=\frac{1}{3}$ and $\frac{1}{2}$, for pseudopotentials of electrons in the first and second LL, and of CF's in the second LL. (b) The total $g(r)$ and "remainder" $g_{\text {diff }}(r)$ curves of the Moore-Read $\nu=\frac{1}{2}$ state; circles mark a fitting linear combination of the curves for Laughlin states.

not deduce the precise cluster size from the short-range behavior of $g(r)$ for the following reasons: (i) $K$ is not a well-defined (conserved) quantum number; (ii) $\nu=\frac{1}{3}$ states occur for all $N$ (not only those divisible by two or three) which means that all clusters cannot have the same $K$; (iii) parameters $\alpha$ and $\beta$ are size-dependent and their extrapolation to large systems is not very reliable based on limited number of $N$-QE systems we are able to diagonalize; (iv) inter-cluster exchange of QE's makes the "independent-cluster" picture only an approximation.

Fortunately, we can use the Moore-Read states (known to be paired ${ }^{7.22}$ ) as a test. Our calculation (for details see Sec. IVC) for $N=14$ and $2 l=25$ gives $\beta_{\mathrm{MR}}=0.336$, somewhat larger than $\beta_{2}$. Hence, we shall assume that $\beta_{K}$ in general underestimates the actual value of $\beta$ in a many-body $K$-clustered state.

For the QE's, we got $\beta=0.319 \approx \beta_{\mathrm{MR}}$ for $N=12$ and $2 l=29\left(\nu=\frac{1}{3}\right)$, and $\beta=0.479$ for $N=14$ and $2 l=25\left(\nu=\frac{1}{2}^{+}\right.$; directly comparable with the MooreRead state). With appropriate reservation, we can hence risk a hypothesis that QE's (on the average) form pairs at $\nu=\frac{1}{3}$ and triplets at $\nu=\frac{1}{2}$ (possible triplet formation might turn out especially intriguing in the context of parafermion statistics ${ }^{25}$ ).

\section{Comparison with Moore-Read state}

The evolution of $g(r)$ when going from the lowest electron LL to the second CF LL (i.e., from $L_{0}$ to CF-LL $\left.L_{1}\right)$ is clear when using a model pseudopotential $V_{\zeta}(\mathcal{R})=\zeta \delta_{\mathcal{R}, 1}+(1-\zeta) \delta_{\mathcal{R}, 3}$. For $\zeta \approx 0$ or 1 , the correlations (avoidance of $\mathcal{R}=1$ or 3 ) are insensitive to $\zeta$, and both Laughlin and QE-QE correlations are accurately reproduced by $V_{0}$ and $V_{1}$, respectively. Modeling correlations among electrons in $\mathrm{LL}_{1}$ (the second LL) is more difficult, because they are very sensitive to the exact form of $V(\mathcal{R})$ at the corresponding $\zeta \sim \frac{1}{2}$. As a result, the $N$-electron Coulomb eigenstates in $\mathrm{LL}_{1}$ are more susceptible to finite-size errors than in $\mathrm{LL}_{0}$ or CF-LL $\mathrm{L}_{1}$. In large systems, a good trial state is only known at $\nu=\frac{1}{2}$ (Moore-Read state), and much less is established about the correlations at $\nu=\frac{1}{3}$. Still, the $g(r)$ curves for electrons in $\mathrm{LL}_{1}$ must certainly fall between the two extreme curves for $\zeta=0$ and 1 (and differ from both of them). This is shown in Fig. 5(a) for both $\nu=\frac{1}{3}$ and $\frac{1}{2}$.

The exact Moore-Read wavefunctions were calculated on a sphere for $N \leq 14$ and $2 l=2 N-3=25$ by diagonalizing a short-range three-body repulsion ${ }^{22}$ In Fig. 5 (a) we only plotted $g(r)$ for $N=14$ because the $N=12$ curve is too close to be easily distinguished. The values of $\alpha=0.602$ and 0.600 for $N=12$ and 14. The $g_{\text {diff }}(r)$, also shown, is positive definite, very different from the QE curves in Fig. 4(b), and rather close to $g_{1}(r)$, where $g_{p}$ describes a Laughlin $\nu=(2 p+1)^{-1}$ state. Assuming $\alpha_{\mathrm{MR}}=\frac{3}{5}$ and expanding $g_{\text {diff }}$ into $g_{1}$ and $g_{2}$ in accordance with Eq. (2) one obtains an approximate formula

$$
g_{\mathrm{MR}}(r) \approx \frac{3}{5} g_{0}(r)+\frac{3}{10} g_{1}(r)+\frac{1}{10} g_{2}(r),
$$

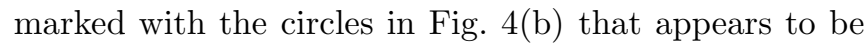
quite accurate (the largest finite-size error is in $g_{2}$ calculated for only $N=8$, while $g_{1}$ is for $N=12$ ).

The fact that $g_{\text {diff }}$ is positive and rather featureless (similar to $g_{p}$ ) for the Moore-Read wavefunction is in contrast with the result for QE's. This difference may indicate that the $\mathrm{QE}$ clusters cannot be understood as literally as Moore-Read pairs. Indeed, even the lack of correlation between the occurrence of $L=0$ ground states (or size of the excitation gap) and the divisibility of $N$ by $K=2$ or 3 precludes such a simple picture. The fact that $g_{\text {diff }}(r \sim 3 \lambda)<0$ could mean that the average relative (with respect to center of mass) angular momentum $\mathcal{R}_{K}$ of the QE clusters is much larger than $\mathcal{R}_{K}^{\min }=\frac{1}{2} K(K-1)$. Certainly, $\mathcal{R}_{K}$ is only conserved for an isolated cluster, but it is possible that the QE clusters are more relaxed due to cluster-cluster interaction than the Moore-Read pairs are. This would make $g_{0}$ underestimate the radius of the actual intra-cluster QE-QE correlation hole, and explain the negative sign of $g_{\text {diff }}$. 


\section{CONCLUSION}

From exact numerical diagonalization on Haldane sphere, we obtained the energy spectra and wavefunctions of up to $N=14$ interacting Laughlin QE's (CF's in the second LL). We identified the series of finite-size liquid ground states with a gap, which extrapolate to the experimentally observed incompressible FQH states at $\nu_{e}=\frac{4}{11}, \frac{3}{8}$, and $\frac{5}{13}$. In these states, we calculated $\mathrm{QE}-\mathrm{QE}$ pair-distribution functions $g(r)$, and showed that they increase as $\sim r^{2}$ at short range and have a pronounced shoulder at a medium range. This behavior supports the idea of QE cluster formation, suggested earlier from the analysis of QE-QE interaction pseudopotential. The $g(r)$ is decomposed into short- and long-range contributions, interpreted as correlations between the QE's from the same or different clusters. The intra-cluster contribution to $g(r)$ is that of a full LL, and the remaining term identified with the inter-cluster $\mathrm{QE}-\mathrm{QE}$ correlations appears to be the same in all three $\nu=\frac{1}{3}, \frac{1}{2}$, and $\frac{2}{3}$ states. The (average) cluster size on the other hand does depend on $\nu$, and we present arguments which suggest that the QE's form pairs at $\nu=\frac{1}{3}$ and triplets at $\nu=\frac{1}{2}$.

The qualitative difference between the $g(r)$ curves obtained here for correlated CF's and those known for the Laughlin and Moore-Read liquids of electrons are another indication that the origin of incompressibility at $\nu_{e}=\frac{4}{11}, \frac{3}{8}$, and $\frac{5}{13}$ is different. Of other hypotheses invoked in literature and mentioned here in the introduction, the broken-symmetry states cannot be excluded by our calculation in spherical geometry. However, we anticipate that the QE's form a liquid (studied in this paper) also in experimental samples, because of the whole series of isotropic ground states with a gap occurring in finite systems of different size.

The authors thank W. Pan, W. Bardyszewski, and L. Bryja for helpful discussions. This work was supported by Grant DE-FG 02-97ER45657 of the Materials Science Program - Basic Energy Sciences of the U.S. Dept. of Energy and Grant 2P03B02424 of the Polish KBN.
${ }^{1}$ W. Pan, H. L. Störmer, D. C. Tsui, L. N. Pfeiffer, K. W. Baldwin, and K. W. West, Phys. Rev. Lett. 90, 016801 (2003); conjugate $\nu=7 / 11$ state was first observed by V.J. Goldman and M. Shayegan [Surface Sci. 229, 10 (1990)].

2 D. C. Tsui, H. L. Störmer, and A. C. Gossard, Phys. Rev. Lett. 48, 1559 (1982).

3 R. B. Laughlin, Phys. Rev. Lett. 50, 1395 (1983).

4 J. K. Jain, Phys. Rev. Lett. 63, 199 (1989).

5 A. Lopez and E. Fradkin, Phys. Rev. B 44, 5246 (1991); B. I. Halperin, P. A. Lee, and N. Read, Phys. Rev. B 47, 7312 (1993).

${ }^{6}$ F. D. M. Haldane, Phys. Rev. Lett. 51, 605 (1983).

7 G. Moore and N. Read, Nucl. Phys. B 360, 362 (1991).

8 P. Sitko, S. N. Yi, K.-S. Yi, and J. J. Quinn, Phys. Rev. Lett. 76, 3396 (1996).

9 A. Wójs and J. J. Quinn, Phys. Rev. B 61, 2846 (2000).

10 S.-Y. Lee, V. W. Scarola, and J. K. Jain, Phys. Rev. Lett. 87, 256803 (2001); Phys. Rev. B 66, 085336 (2002).

11 S. S. Mandal and J. K. Jain, Phys. Rev. B 66, 155302 (2002).

12 A. Wójs, K.-S. Yi, and J. J. Quinn, Phys. Rev. B 69, $205322(2004)$.

13 C.-C. Chang and J. K. Jain, Phys. Rev. Lett. 92, 196806 (2004).

14 M. R. Peterson and J. K. Jain, Phys. Rev. Lett. 93, 046402
(2004); J. H. Smet, Nature 422, 391 (2003).

15 M. Flohr and K. Osterloh, Phys. Rev. B 67, 235316 (2003).

16 A. Wójs, K.-S. Yi, and J. J. Quinn, Acta Phys. Pol. A 103, 517 (2003); J. J. Quinn, A. Wójs, and K.-S. Yi, Phys. Lett. A 318, 152 (2003).

17 N. Shibata and D. Yoshioka, J. Phys. Soc. Jpn. 72, 664 (2003); J. Phys. Soc. Jpn. 73, 1 (2004); J. Phys. Soc. Jpn. 73, 2169 (2004).

18 F. D. M. Haldane and E. H. Rezayi, Phys. Rev. Lett. 54, 237 (1985).

19 A. Wójs and J. J. Quinn, Philos. Mag. B 80, 1405 (2000); Acta Phys. Pol. A 96, 593 (1999); J. J. Quinn and A. Wójs, J. Phys.: Condens. Matter 12, R265 (2000).

20 M. O. Goerbig, P. Lederer, and C. M. Smith, Phys. Rev. Lett. 93, 216802 (2004); Phys. Rev. B 69, 155324 (2004).

21 F. D. M. Haldane, The Quantum Hall Effect, edited by R. E. Prange and S. M. Girvin, (Springer-Verlag, New York, 1987), chapter 8, pp. 303-352.

22 A. Wójs and J. J. Quinn, Phys. Rev. B 71, 045324 (2005).

${ }^{23}$ K. Musaelian and R. Joynt, J. Phys: Condens. Matter 8, L105 (1996); O. Ciftja and C. Wexler, Phys. Rev. B 65, 045306 (2002); Phys. Rev. B 65, 205307 (2002).

24 B. Jancovici, Phys. Rev. Lett. 46, 386 (1981).

25 N. Read and E. Rezayi, Phys. Rev. B 59, 8084 (1999). 